\title{
The frequency and distribution of caries in the mediaeval population of Bijelo Brdo in Croatia (10th-11th century)
}

\author{
Vodanović Marin $^{a, *}$, Brkić Hrvoje ${ }^{b}$, Šlaus Marioc ${ }^{c}$ Demo Željko \\ a Department of Physiology, School of Dental Medicine, \\ University of Zagreb, Gunduliceva 5, 10000 Zagreb, Croatia \\ ${ }^{\mathrm{b}}$ Department of Dental Anthropology, School of Dental Medicine, University of Zagreb, Croatia \\ 'Department of Archaeology, Croatian Academy of Sciences and Arts, Zagreb, Croatia \\ 'Museum of Archaeology, Zagreb, Croatia
}

Accepted 16 November 2004

\section{KEYWORDS \\ Archaeology; Medieval; \\ Dental caries; \\ Epidemiology; \\ Croatia}

\begin{abstract}
Summary Reconstruction of the life of ancient peoples can be accomplished by studying their dental remains. The further we go into the past, the greater the importance of dental remains for answering a wide spectre of questions related to the life of a particular community. The dental system is a valuable source of information on the type of food, illnesses, and social stratification within a community.

The purpose of this paper was to determine the frequency, distribution, and characteristics of dental caries in the mediaeval population of Bijelo Brdo in Croatia.

The analysed sample consisted of the dental remains of 85 individuals with the total of 1064 teeth. The majority (979 or $92.0 \%$ ) of the teeth belonged to the permanent dentition, and this data set was analysed in this report. The frequency of antemortem tooth loss in the sample was $6.7 \%$, the frequency of caries $-9.5 \%$. The most frequent recorded caries were interproximal (3.9\%), followed by occlusal $(2.9 \%)$, and buccal/lingual (1.3\%).

This research showed that the frequency and distribution of dental caries in the early mediaeval population from Bijelo Brdo is very similar to that of other European populations of the same socio-economic status during the same historic period. Chronological changes in the localisation of caries in populations that inhabited continental Croatia during the mediaeval period indicate a gradual reduction of interproximal caries and an increase of occlusal, buccal, and lingual lesions. These data suggest a change of diet with softer foods becoming more available in the younger time periods.
\end{abstract}

(C) 2004 Elsevier Ltd. All rights reserved.

\footnotetext{
* Corresponding author. Tel.: +385 912548554.

E-mail address: mav@net.hr (M. Vodanović).
} 


\section{Introduction}

The frequency and distribution of dental caries in ancient populations has been the subject of study for a long time. That enables us to continuously follow their economic, social, and cultural progress through different historic periods. ${ }^{1-4}$

Teeth are one of the most enduring physical evidences of existence of an individual after death. As such, they provide good material for palaeodental research for two reasons. ${ }^{5}$ Firstly, teeth have an extremely great resistance to postmortem damages and can retain their original shape for a very long time regardless of their postmortem environment. Secondly, as dental procedures in the past (restoration and oral surgery) were non-existent or rare the epidemiology of caries can be studied in its original shape. ${ }^{6}$

We can find out a great deal about our ancestors based on teeth and teeth pathology. The system and consistency of consumed food determines the types of micro-organisms that are found in the oral cavity, as well as the nature of the biomechanical forces that affected teeth and the jawbone. Diagnosis and interpretation of dental illnesses in palaeodemographic contexts are important steps in the attempt to reconstruct past lives. These data tell us what people ate and how they lived. ${ }^{7,8}$

The recent foundation of the Osteological collection of the Croatian academy of sciences and arts, which houses the remains of approximately 5000 individuals from 51 archaeological sites in Croatia dating form $5000 \mathrm{BC}$ to 18th century has greatly facilitated the analysis of dental disease in archaeological populations. ${ }^{4}$ The purpose of this paper is to determine the frequency and distribution of caries in the mediaeval population from the Bijelo Brdo site in Croatia, and to compare the results with data from several large mediaeval sites in continental Croatia, as well as with several mediaeval European populations. The analysed sites from Croatia include Privlaka, Stenjevec, Vinkovci, Djakovo, Djelekovec, Scitarjevo, and Lobor. ${ }^{4}$ The analysed European populations are La-Selvicciola from Italy, ${ }^{9}$ Whithorn from Scotland, ${ }^{6}$ and Iznik from Turkey. ${ }^{2}$

\section{Materials and methods}

The research was carried out on the skeletal remains of 85 individuals excavated at the end of 19 th century and the beginning of 20th century, from the early mediaeval (10th-11th centuries) cemetery at the archaeological site Bijelo Brdo near Osijek. The samples were stored in the Museum of Archaeology in Zagreb.
In this analysis all available skulls were analysed, regardless of the level of damage. The state of preservation varied from completely preserved skulls with complete mandibles, to cases where only small fragments of the maxilla or mandible were preserved (Table 1). The following levels of preservation of the maxilla and mandible were noted in the series:

- Level 1 - indicating preservation of both maxilla and mandible and preservation of more than $50 \%$ of alveolar bone,

- Level 2 - indicating preservation of both maxilla and mandible but with preservation of less than $50 \%$ of alveolar bone,

- Level 3 - indicating preservation of only the maxilla or the mandible and preservation of more than $50 \%$ of alveolar bone,

- Level 4 - indicating preservation of only the maxilla or the mandible and preservation of less than $50 \%$ of alveolar bone.

Selection of only well preserved remains could result in an unrealistically low frequency of caries. The reason for this is that bones weakened by various pathologies during an individual's life were more likely to suffer postmortem damage than bones of a healthy individuals. ${ }^{6}$ Besides mechanical damage, some of the skeletal remains exhibited postmortem erosion. This type of damage is most often the result of collagen loss which results in a gradual loss of bone mass, dental cement, and dentine. ${ }^{10}$

Age at death was determined by dental development in cases with deciduous and mixed dentition, and by cranial suture fusion and teeth abrasion in adults. ${ }^{11}$

Age classification was carried out according to the criteria of Watt et al. ${ }^{6}$ Samples were classified into the following groups:

\begin{tabular}{ll}
\hline $6-12$ years & Juvenile (mixed dentition) \\
$13-20$ years & Adolescent \\
$21-25$ years & $\begin{array}{l}\text { Young adults (at least one third } \\
\text { molar erupted) }\end{array}$ \\
$26-35$ years & Adults \\
$36-45$ years & Mature adults \\
$46+$ & Older adults \\
\hline
\end{tabular}

Table 1 Preservation of the skeletal remains.

\begin{tabular}{llrl}
\hline $\begin{array}{l}\text { Level of } \\
\text { preservation }\end{array}$ & $\begin{array}{l}\text { Number of } \\
\text { individuals (\%) }\end{array}$ & $\begin{array}{l}\text { Number of } \\
\text { upper jaws }\end{array}$ & $\begin{array}{l}\text { Number of } \\
\text { lower jaws }\end{array}$ \\
\hline 1 & $39(46)$ & 39 & 39 \\
2 & $8(9)$ & 8 & 8 \\
3 & $30(35)$ & 13 & 17 \\
4 & $8(9)$ & 3 & 5 \\
Total & $85(100)$ & 63 & 69 \\
\hline
\end{tabular}


Table 2 Teeth present, teeth lost ante- and postmortem.

\begin{tabular}{lcccrr}
\hline Age group & $\begin{array}{l}\text { Number of } \\
\text { individuals }\end{array}$ & $\begin{array}{l}\text { Teeth } \\
\text { present }\end{array}$ & $\begin{array}{l}\text { Teeth present } \\
+ \text { AM + PM loss }\end{array}$ & $\begin{array}{l}\text { Antemortem } \\
\text { loss (\%) }\end{array}$ & $\begin{array}{r}\text { Postmortem } \\
\text { loss (\%) }\end{array}$ \\
\hline $6-12$ & 7 & 56 & 74 & $0(0.0)$ & $0(0.0)$ \\
$13-20$ & 5 & 57 & 64 & $19(3.5)$ & $7(10.9)$ \\
$21-25$ & 30 & 361 & 538 & $7(4.8)$ & $158(29.4)$ \\
$26-35$ & 8 & 104 & 145 & $47(10.5)$ & $34(23.4)$ \\
$36-45$ & 23 & 258 & 449 & $26(11.9)$ & $144(32.1)$ \\
$46+$ & 8 & 143 & 218 & $99(6.7)$ & $49(22.5)$ \\
Total & 81 & 979 & 1488 & & $410(27.6)$ \\
\hline
\end{tabular}

Tooth loss was classified as ante- or postmortem. Teeth were considered lost postmortem if there was clear evidence of alveolar socket.

Caries were diagnosed macroscopically under a bright light, with the help of a dental probe. A lesion was considered a caries if there was a clear defect in tooth tissue. Colour changes of the enamel were not considered caries unless there was cavitation underneath. The number of caries, as well as their location (occlusal, mesial, distal, buccal, and lingual) was noted. Caries frequencies, frequencies of carious lesions on the various tooth surfaces, and the skeletal root caries index were calculated. The skeletal root caries index was calculated using the formula: total number of carious root surfaces/total number of root surfaces in erupted teeth $\times 100$.

Estimation of skeletal age at death and caries diagnosis was carried out by one observer (MV). The statistical significance of the recorded values was tested with the chi-square test.

Our findings were compared with findings of other authors who investigated the frequency and distribution of caries in medieval populations in continental Croatia, ${ }^{4}$ Turkey, ${ }^{2}$ Scotland ${ }^{6}$ and Italy 9 .

\section{Results}

The analysed sample consisted of the skeletal remains of 85 individuals that had a total of 1064 teeth. The majority ( 979 teeth or $92 \%$ ) belonged to the permanent dentition while 85 teeth (8\%) belonged to the deciduous dentition. Four individuals were under 6 years of age and therefore had only deciduous dentition. In this analysis only permanent teeth were analysed.

Table 2 shows the number of teeth analysed, as well as the frequencies of antemortem and postmortem tooth loss in the analysed age groups. The frequency of antemortem tooth loss was significantly higher $(P<0.001)$ in the three older age categories (26 to $46+$ years) when compared to the younger age groups (6-25 years). No antemortem tooth loss was noted in the two youngest age categories. In the oldest age category $11.9 \%$ of teeth were lost antemortally. The total frequency of antemortem tooth loss for the sample was $6.7 \%$. Postmortem tooth loss was significantly higher $(27.6 \%)$ and uniformly distributed in all age groups. Incisors were the teeth most frequently lost postmortem while canines had the highest rate of preservation.

More than $46 \%$ of the analysed individuals with permanent dentition have at least one carious lesion. As shown in Table 3, the number of individuals that exhibited at least one carious lesion was significantly higher $(P<0.001)$ in the older age groups ( 26 to $46+$ years) then in the younger (6-25 years).

Table 4 shows the frequencies of carious lesions in the analysed age categories according to tooth type. The frequency of carious lesions clearly increased with age. The lowest frequency was noted in the youngest age group (1.8\%), the highest in the oldest (14.0\%). The total frequency of carious lesions in the sample was $9.5 \%$. The difference between carious frequencies in the younger $(6.8 \%)$ and older age groups $(12.1 \%)$ was significant $(P<0.05)$.

While in the oldest age group all types of teeth were affected with caries, only one carious tooth was noted in the youngest age group - the first maxillary molar. Older age categories exhibited both a higher frequency of carious lesions and a higher number of tooth types affected. Furthermore, a trend towards a more mesial location of the lesions from molars, to premolars, to canines,

Table 3 Individuals with caries experience.

\begin{tabular}{lcc}
\hline Age group & $\begin{array}{c}\text { Number of } \\
\text { individuals }\end{array}$ & $\begin{array}{l}\text { Number and } \\
\text { percentage of } \\
\text { individuals with caries }\end{array}$ \\
\hline $6-12$ & 7 & $1(14.3)$ \\
$13-20$ & 5 & $2(40.0)$ \\
$21-25$ & 30 & $7(23.3)$ \\
$26-35$ & 8 & $6(75.0)$ \\
$36-45$ & 23 & $16(69.6)$ \\
$46+$ & 8 & $6(75.0)$ \\
Total & 81 & $38(46.9)$ \\
\hline
\end{tabular}


Table 4 Caries prevalence.

\begin{tabular}{|c|c|c|c|c|c|c|c|c|c|c|c|c|c|c|c|c|c|c|c|c|c|c|c|c|c|}
\hline \multirow[t]{2}{*}{ Age group } & \multicolumn{12}{|c|}{ Upper jaw } & \multicolumn{13}{|c|}{ Lower jaw } \\
\hline & 11 & 12 & $11+12$ & c & P1 & P2 & $\mathrm{P} 1+\mathrm{P} 2$ & M1 & M2 & M3 & $\begin{array}{l}M 1+ \\
M 2+ \\
M 3\end{array}$ & $\begin{array}{l}\text { Upper } \\
\text { jaw } \\
\text { total }\end{array}$ & 11 & 12 & $11+12$ & $\mathrm{C}$ & P1 & P2 & $\mathrm{P} 1+\mathrm{P} 2$ & M1 & M2 & M3 & $\begin{array}{l}M 1+ \\
M 2+ \\
M 3\end{array}$ & $\begin{array}{l}\text { Lower } \\
\text { jaw } \\
\text { total }\end{array}$ & Total \\
\hline \multicolumn{26}{|l|}{$6-12$} \\
\hline Number of teeth present & 6 & 3 & 9 & 2 & 0 & 0 & 0 & 13 & 0 & 0 & 13 & 24 & 8 & 8 & 16 & 1 & 0 & 0 & 0 & 13 & 2 & 0 & 15 & 32 & 56 \\
\hline Number of carious teeth & - & - & - & - & - & - & - & 1 & - & - & 1 & 1 & - & - & - & - & - & - & - & - & - & - & - & 0 & 1 \\
\hline$\%$ of carious teeth & - & - & - & - & - & - & - & 7.7 & - & - & 7.7 & 4.2 & - & - & - & - & - & - & - & - & - & - & - & 0.0 & 1.8 \\
\hline \multicolumn{26}{|l|}{$13-20$} \\
\hline Number of teeth present & 5 & 5 & 10 & 3 & 5 & 4 & 9 & 5 & 2 & 0 & 7 & 29 & 0 & 4 & 4 & 4 & 5 & 5 & 10 & 5 & 5 & 0 & 10 & 28 & 57 \\
\hline Number of carious teeth & - & - & - & - & - & - & - & - & 1 & - & 1 & 1 & - & - & - & - & - & 1 & 1 & - & 1 & - & 1 & 2 & 3 \\
\hline$\%$ of carious teeth & - & - & - & - & - & - & - & - & 50.0 & - & 14.3 & 3.4 & - & - & - & - & - & 20.0 & 10.0 & - & 20.0 & - & 10.0 & 7.1 & 5.3 \\
\hline \multicolumn{26}{|l|}{$21-25$} \\
\hline Number of teeth present & 10 & 20 & 30 & 22 & 22 & 21 & 43 & 26 & 29 & 15 & 70 & 165 & 16 & 20 & 36 & 27 & 28 & 28 & 56 & 26 & 32 & 19 & 77 & 196 & 361 \\
\hline Number of carious teeth & - & - & - & 2 & - & 1 & 1 & 5 & 5 & 3 & 13 & 16 & - & - & - & - & 1 & - & 1 & 3 & 6 & 2 & 11 & 12 & 28 \\
\hline$\%$ of carious teeth & - & - & - & 9.1 & - & 4.8 & 2.3 & 19.2 & 17.2 & 20.0 & 18.6 & 9.7 & - & - & - & - & 3.6 & - & 1.8 & 11.5 & 18.8 & 10.5 & 14.3 & 6.1 & 7.8 \\
\hline \multicolumn{26}{|l|}{$26-35$} \\
\hline Number of teeth present & 5 & 8 & 13 & 8 & 7 & 10 & 17 & 10 & 6 & 1 & 17 & 55 & 3 & 4 & 7 & 5 & 7 & 8 & 15 & 8 & 9 & 5 & 22 & 49 & 104 \\
\hline Number of carious teeth & - & - & - & 1 & - & - & - & 1 & 1 & 1 & 3 & 4 & - & - & - & - & - & - & - & - & - & 2 & 2 & 2 & 6 \\
\hline$\%$ of carious teeth & - & - & - & 12.5 & - & - & - & 10.0 & 16.7 & 100.0 & 17.6 & 7.3 & - & - & - & - & - & - & - & - & - & 40.0 & 9.1 & 4.1 & 5.8 \\
\hline \multicolumn{26}{|l|}{$36-45$} \\
\hline Number of teeth present & 5 & 10 & 15 & 15 & 18 & 18 & 36 & 16 & 17 & 7 & 40 & 106 & 17 & 16 & 33 & 18 & 21 & 22 & 43 & 21 & 22 & 15 & 58 & 152 & 258 \\
\hline Number of carious teeth & - & - & - & - & 1 & 2 & 3 & 2 & 3 & 1 & 6 & 9 & - & - & - & - & - & 2 & 2 & 6 & 10 & 8 & 24 & 26 & 35 \\
\hline$\%$ of carious teeth & - & - & - & - & 5.6 & 11.1 & 8.3 & 12.5 & 17.6 & 14.3 & 15.0 & 8.5 & - & - & - & - & - & 9.1 & 4.7 & 28.6 & 45.5 & 53.3 & 41.4 & 17.1 & 13.6 \\
\hline \multicolumn{26}{|l|}{$46+$} \\
\hline Number of teeth present & 7 & 8 & 15 & 10 & 10 & 10 & 20 & 9 & 7 & 3 & 19 & 64 & 7 & 11 & 18 & 13 & 12 & 8 & 20 & 7 & 11 & 10 & 28 & 79 & 143 \\
\hline Number of carious teeth & 1 & 1 & 2 & - & 2 & 2 & 4 & 2 & 3 & 1 & 6 & 12 & - & 1 & 1 & 1 & 1 & - & 1 & 2 & 2 & 1 & 5 & 8 & 20 \\
\hline$\%$ of carious teeth & 14.3 & 12.5 & 13.3 & - & 20.0 & 20.0 & 20.0 & 22.2 & 42.9 & 33.3 & 31.6 & 18.8 & - & 9.1 & 5.6 & 7.7 & 8.3 & - & 5.0 & 28.6 & 18.2 & 10.0 & 17.9 & 10.1 & 14.0 \\
\hline \multicolumn{26}{|l|}{ Total } \\
\hline Number of teeth present & 38 & 54 & 92 & 60 & 62 & 63 & 125 & 79 & 61 & 26 & 166 & 443 & 51 & 63 & 114 & 68 & 73 & 71 & 144 & 80 & 81 & 49 & 210 & 536 & 979 \\
\hline Number of carious teeth & 1 & 1 & 2 & 3 & 3 & 5 & 8 & 11 & 13 & 6 & 30 & 43 & - & 1 & 1 & 1 & 2 & 3 & 5 & 11 & 19 & 13 & 43 & 50 & 93 \\
\hline$\%$ of carious teeth & 2.6 & 1.9 & 2.2 & 5.0 & 4.8 & 7.9 & 6.4 & 13.9 & 21.3 & 23.1 & 18.1 & 9.7 & - & 1.6 & 0.9 & 1.5 & 2.7 & 4.2 & 3.5 & 13.8 & 23.5 & 26.5 & 20.5 & 9.3 & 9.5 \\
\hline
\end{tabular}


Table 5 Location of caries.

\begin{tabular}{|c|c|c|c|c|c|c|c|c|c|c|c|c|c|c|}
\hline Age group & $\begin{array}{l}\text { Number } \\
\text { of } \\
\text { occlusal } \\
\text { surfaces }\end{array}$ & $\begin{array}{l}\text { Number } \\
\text { of } \\
\text { occlusal } \\
\text { caries } \\
\text { (\%) }\end{array}$ & $\begin{array}{l}\text { Number } \\
\text { of } \\
\text { mesial } \\
\text { surfaces }\end{array}$ & $\begin{array}{l}\text { Number } \\
\text { of } \\
\text { mesial } \\
\text { caries } \\
\text { (\%) }\end{array}$ & $\begin{array}{l}\text { Number } \\
\text { of } \\
\text { distal } \\
\text { surfaces }\end{array}$ & $\begin{array}{l}\text { Number } \\
\text { of } \\
\text { distal } \\
\text { caries } \\
\text { (\%) }\end{array}$ & $\begin{array}{l}\text { Number } \\
\text { of } \\
\text { approximal } \\
\text { surfaces }\end{array}$ & $\begin{array}{l}\text { Number } \\
\text { of } \\
\text { approximal } \\
\text { caries } \\
\text { (\%) }\end{array}$ & $\begin{array}{l}\text { Number } \\
\text { of } \\
\text { buccal (B) } \\
\text { surfaces }\end{array}$ & $\begin{array}{l}\text { Number } \\
\text { of } \\
\text { buccal } \\
\text { caries } \\
(\%)\end{array}$ & $\begin{array}{l}\text { Number } \\
\text { of } \\
\text { lingual (L) } \\
\text { surfaces }\end{array}$ & $\begin{array}{l}\text { Number } \\
\text { of } \\
\text { lingual } \\
\text { caries } \\
(\%)\end{array}$ & $\begin{array}{l}\text { Number } \\
\text { of } \\
B+L \\
\text { surfaces }\end{array}$ & $\begin{array}{l}\text { Number } \\
\text { of } \\
B+L \\
\text { caries } \\
(\%)\end{array}$ \\
\hline \multicolumn{15}{|l|}{ Upper jaw } \\
\hline $6-12$ & 13 & 1 (7.7) & 24 & $0(0.0)$ & 24 & $0(0.0)$ & 48 & $0(0.0)$ & 24 & $0(0.0)$ & 24 & $0(0.0)$ & 48 & $0(0.0)$ \\
\hline $13-20$ & 16 & $1(6.3)$ & 29 & $0(0.0)$ & 29 & $0(0.0)$ & 58 & $0(0.0)$ & 29 & $0(0.0)$ & 29 & $0(0.0)$ & 58 & $0(0.0)$ \\
\hline $21-25$ & 113 & $10(8.8)$ & 165 & $1(0.6)$ & 165 & $5(3.0)$ & 330 & $6(1.8)$ & 165 & $1(0.6)$ & 165 & $0(0.0)$ & 330 & $18(0.3)$ \\
\hline $26-35$ & 34 & $0(0.0)$ & 55 & $2(3.6)$ & 55 & $2(3.6)$ & 110 & $4(3.6)$ & 55 & $0(0.0)$ & 55 & $0(0.0)$ & 110 & $0(0.0)$ \\
\hline $36-45$ & 76 & $0(0.0)$ & 106 & $5(4.7)$ & 106 & $5(4.7)$ & 212 & $10(4.7)$ & 106 & $1(0.9)$ & 106 & $0(0.0)$ & 212 & $1(0.5)$ \\
\hline $46+$ & 39 & $0(0.0)$ & 64 & $8(12.5)$ & 64 & $4(6.3)$ & 128 & $12(9.4)$ & 64 & $0(0.0)$ & 64 & $0(0.0)$ & 128 & $0(0.0)$ \\
\hline Total & 291 & $12(4.1)$ & 443 & $16(3.6)$ & 443 & $16(3.6)$ & 886 & $32(3.6)$ & 443 & $2(0.5)$ & 443 & $0(0.0)$ & 886 & $2(0.2)$ \\
\hline \multicolumn{15}{|l|}{ Lower jaw } \\
\hline $6-12$ & 15 & $0(0.0)$ & 32 & $0(0.0)$ & 32 & $1(3.1)$ & 64 & 1 (1.6) & 32 & $1(3.1)$ & 32 & $0(0.0)$ & 64 & $1(1.6)$ \\
\hline $13-20$ & 20 & $0(0.0)$ & 28 & $0(0.0)$ & 28 & $1(3.6)$ & 56 & $1(1.8)$ & 28 & $2(7.1)$ & 28 & $1(3.6)$ & 56 & $3(5.4)$ \\
\hline $21-25$ & 133 & $3(2.3)$ & 196 & $5(2.6)$ & 196 & $6(3.1)$ & 392 & $11(2.8)$ & 196 & $11(5.6)$ & 196 & $0(0.0)$ & 392 & $11(2.8)$ \\
\hline $26-35$ & 37 & $1(2.7)$ & 49 & $1(2.0)$ & 49 & $1(2.0)$ & 98 & $2(2.0)$ & 49 & $0(0.0)$ & 49 & $0(0.0)$ & 98 & $0(0.0)$ \\
\hline $36-45$ & 101 & $2(2.0)$ & 152 & $13(8.6)$ & 152 & $8(5.3)$ & 304 & $21(6.9)$ & 152 & $5(3.3)$ & 152 & $2(1.3)$ & 304 & $7(2.3)$ \\
\hline $46+$ & 48 & $1(2.1)$ & 79 & $3(3.8)$ & 79 & $5(6.3)$ & 158 & $8(5.1)$ & 79 & $1(1.3)$ & 79 & $0(0.0)$ & 158 & $1(0.6)$ \\
\hline Total & 354 & $7(2.0)$ & 536 & $22(4.1)$ & 536 & $22(4.1)$ & 1072 & $44(4.1)$ & 536 & $20(3.7)$ & 536 & $3(0.6)$ & 1072 & $23(2.1)$ \\
\hline \multicolumn{15}{|c|}{ Upper jaw + lower jaw } \\
\hline $6-12$ & 28 & $1(3.6)$ & 56 & $0(0.0)$ & 56 & $1(1.8)$ & 112 & $1(0.9)$ & 56 & $1(1.8)$ & 56 & $0(0.0)$ & 112 & $1(0.9)$ \\
\hline $13-20$ & 36 & $1(2.8)$ & 57 & $0(0.0)$ & 57 & $1(1.8)$ & 114 & $1(0.9)$ & 57 & $2(3.5)$ & 57 & $1(1.8)$ & 114 & $3(2.6)$ \\
\hline $21-25$ & 246 & $13(5.3)$ & 361 & $6(1.7)$ & 361 & $11(3.0)$ & 722 & $17(2.4)$ & 361 & $12(3.3)$ & 361 & $0(0.0)$ & 722 & $12(1.7)$ \\
\hline $26-35$ & 71 & 1 (1.4) & 104 & $3(2.9)$ & 104 & $3(2.9)$ & 208 & $6(2.9)$ & 104 & $0(0.0)$ & 104 & $0(0.0)$ & 208 & $0(0.0)$ \\
\hline $36-45$ & 177 & $2(1.1)$ & 258 & $18(7.0)$ & 258 & $13(5.0)$ & 516 & $31(6.0)$ & 258 & $6(2.3)$ & 258 & $2(0.8)$ & 516 & $8(1.6)$ \\
\hline $46+$ & 87 & $1(1.1)$ & 143 & $11(7.7)$ & 143 & $9(6.3)$ & 286 & $20(7.0)$ & 143 & $1(0.7)$ & 143 & $0(0.0)$ & 286 & $1(0.3)$ \\
\hline Total & 645 & $19(2.9)$ & 979 & 38 (3.9) & 979 & 38 (3.9) & 1958 & $76(3.9)$ & 979 & $22(2.2)$ & 979 & $3(0.3)$ & 1958 & $25(1.3)$ \\
\hline
\end{tabular}


Table 6 Number of carious surfaces per carious tooth.

\begin{tabular}{|c|c|c|c|}
\hline & $\begin{array}{l}\text { Number of } \\
\text { carious } \\
\text { teeth }\end{array}$ & $\begin{array}{l}\text { Number } \\
\text { of } \\
\text { carious } \\
\text { surfaces }\end{array}$ & $\begin{array}{l}\text { Average } \\
\text { number of } \\
\text { carious } \\
\text { surfaces per } \\
\text { carious tooth }\end{array}$ \\
\hline \multicolumn{4}{|l|}{ Anterior teeth } \\
\hline Maxillary & 5 & 6 & 1.2 \\
\hline Mandibular & 2 & 2 & 1.0 \\
\hline \multicolumn{4}{|l|}{ Posterior teeth } \\
\hline Maxillary & 38 & 40 & 1.1 \\
\hline Mandibular & 48 & 72 & 1.5 \\
\hline $\begin{array}{l}\text { All maxillary } \\
\text { teeth }\end{array}$ & 43 & 46 & 1.1 \\
\hline $\begin{array}{l}\text { All mandibular } \\
\text { teeth }\end{array}$ & 50 & 74 & 1.5 \\
\hline Total & 93 & 120 & 1.3 \\
\hline
\end{tabular}

and incisors was evident in the older age categories. In the younger age groups caries was found mostly on the posterior teeth, whereas in the older age groups caries progressed from posterior to anterior teeth, so that in some cases posterior and anterior teeth were affected by caries almost equally.

Central incisors exhibited the lowest frequency of caries $(1.1 \%)$, while the highest frequency was recorded in third molars (25.3\%). Analysis by tooth type showed that incisors exhibited carious lesions in $1.5 \%$, canines in $3.1 \%$, premolars in $4.8 \%$, and molars in $19.4 \%$. These differences were significant $(P<0.001)$. The same pattern was noted when maxillary and mandibular teeth were analysed independently. In the maxilla caries were noted in $2.2 \%$ of incisors, $5.0 \%$ of canines, $6.4 \%$ of premolars, and $18.1 \%$ of all molars. These differences were significant at the $P<0.05$ level. Mandibular teeth exhibited the following frequencies: incisors $0.9 \%$, canines $1.5 \%$, premolars $3.5 \%$, and molars $20.5 \%$. These differences were also significant $(P<0.001)$. The difference between caries frequencies in the maxilla $(9.7 \%)$ and the mandible $(9.3 \%)$ was not statistically significant.

Significant differences in caries frequencies were, however, noted between anterior and posterior teeth regardless of whether the maxilla and mandible were observed together $(P<0.001)$, or independently (maxilla $P<0.05$, mandible $P<$ 0.001).

Table 5 shows the frequency and distribution of caries with respect to the tooth surface on which the caries was located. The number of tooth surfaces affected with caries increased with age; however no significant differences were noted among the age groups. There was, however, a significant difference $(P<0.001)$ in frequencies of different types of carious lesions. Interproximal caries were most frequent $(3.9 \%)$, followed by occlusal $(2.9 \%)$, and buccal/lingual (1.3\%). The most frequent carious lesions in the maxilla were interproximal (69.6\%), followed by occlusal $(26.1 \%)$, and buccal lesions (4.3\%). Lingual lesions were not noticed. The most frequent carious lesions in the mandible were interproximal lesions (59.5\%), followed by buccal $(27.0 \%)$, occlusal $(9.5 \%)$, and lingual lesions (4.1\%).

Caries were recorded in $2.9 \%$ of all occlusal surfaces. Lesions were more frequent in the maxilla $(4.1 \%)$ than in the mandible $(2.0 \%)$. A significant difference $(P<0.05)$ in the frequencies of occlusal caries in the maxilla was noted between the older and younger age groups.

Interproximal caries were recorded in $76 / 1985$ (3.9\%) interproximal surfaces. Differences in the frequencies of interproximal were statistically significant $(P<0.05 \%)$, and increased with age, which can best be seen in the maxilla. In the youngest age groups interproximal caries were found in $0.9 \%$ of all interproximal surfaces. The frequency of interproximal caries in the oldest age groups was 7.0\%. Mesial and distal surfaces were equally affected: $3.6 \%$ of the lesions were noted in the maxilla, and $4.1 \%$ in the mandible. There was a significant difference $(P<0.05 \%)$ among particular age groups with respect to frequencies of mesial caries.

There was a significant difference $(P<0.05 \%)$ between the frequencies of buccal caries in the maxilla $(0.5 \%)$ and mandible $(3.7 \%)$. Only $0.3 \%$ of lingual, and $2.2 \%$ of all buccal surfaces were carious.

Table 6 shows the average number of carious surfaces per carious tooth. The average number of carious surfaces was lower in anterior than posterior teeth, and was higher in the mandible. The average number for all teeth was 1.3 carious surfaces per carious tooth.

The skeletal root caries index $(\mathrm{SRCl})$ is presented in Table 7. The total SRCI was 0.9, and increased with age. The older age groups (26 to $46+$ years) exhibited higher $\mathrm{SRCl}$ than the younger $(6-25$ years) age groups, but the difference was not statistically significant. Anterior teeth exhibited a lower SRCI than posterior teeth. SRCl of anterior maxillary teeth was higher than the SRCI of anterior mandibular teeth. In contrast, the SRCl of posterior mandibular teeth was higher than the SRCl of the maxillary posterior teeth. Mandibular teeth exhibited a higher SRCI than maxillary teeth. There was a significant difference $(P<0.001)$ between the frequencies of carious tooth surfaces and dental roots between maxillary $(0.3 \%)$ and mandibular $(2.3 \%)$ teeth. A significant difference $(P<0.05)$ was also 
Table 7 Skeletal root caries index (SRCI).

\begin{tabular}{|c|c|c|c|c|c|c|c|c|c|c|c|c|c|}
\hline \multirow[t]{2}{*}{ Age group } & \multicolumn{6}{|c|}{ Anterior teeth } & \multicolumn{7}{|c|}{ Posterior teeth } \\
\hline & $\begin{array}{l}\text { Number } \\
\text { of } \\
\text { teeth }\end{array}$ & $\begin{array}{l}\text { Number } \\
\text { of } \\
\text { root } \\
\text { surfaces }\end{array}$ & $\begin{array}{l}\text { Carious } \\
\text { approximal } \\
\text { root } \\
\text { surfaces }\end{array}$ & $\begin{array}{l}\text { Carious } \\
\text { buccal/lingual } \\
\text { root } \\
\text { surfaces }\end{array}$ & $\begin{array}{l}\text { Total of } \\
\text { carious } \\
\text { root } \\
\text { surfaces }\end{array}$ & $\begin{array}{l}\text { Anterior } \\
\text { SRCI }\end{array}$ & $\begin{array}{l}\text { Number } \\
\text { of } \\
\text { teeth }\end{array}$ & $\begin{array}{l}\text { Number } \\
\text { of } \\
\text { root } \\
\text { surfaces }\end{array}$ & $\begin{array}{l}\text { Carious } \\
\text { approximal } \\
\text { root } \\
\text { surfaces }\end{array}$ & $\begin{array}{l}\text { Carious } \\
\text { buccal/lingual } \\
\text { root } \\
\text { surfaces }\end{array}$ & $\begin{array}{l}\text { Total } \\
\text { of } \\
\text { carious } \\
\text { root } \\
\text { surfaces }\end{array}$ & $\begin{array}{l}\text { Posterior } \\
\mathrm{SRCl}\end{array}$ & $\begin{array}{l}\text { Anterior } \\
+ \text { posterior } \\
\text { SRCI }\end{array}$ \\
\hline \multicolumn{14}{|l|}{ Upper jaw } \\
\hline $6-12$ & 11 & 44 & 0 & 0 & 0 & 0.0 & 13 & 52 & 0 & 0 & 0 & 0.0 & 0.0 \\
\hline $13-20$ & 13 & 52 & 0 & 0 & 0 & 0.0 & 16 & 64 & 0 & 0 & 0 & 0.0 & 0.0 \\
\hline $21-25$ & 52 & 208 & 0 & 0 & 0 & 0.0 & 113 & 452 & 1 & 0 & 1 & 0.2 & 0.2 \\
\hline $26-35$ & 21 & 84 & 0 & 1 & 1 & 1.2 & 34 & 136 & 0 & 0 & 0 & 0.0 & 0.5 \\
\hline $36-45$ & 30 & 120 & 0 & 0 & 0 & 0.0 & 76 & 304 & 1 & 1 & 2 & 0.7 & 0.5 \\
\hline $46+$ & 25 & 100 & 0 & 0 & 0 & 0.0 & 39 & 156 & 0 & 0 & 0 & 0.0 & 0.0 \\
\hline Total & 152 & 608 & 0 & 1 & 1 & 0.2 & 291 & 1164 & 2 & 1 & 3 & 0.3 & 0.2 \\
\hline \multicolumn{14}{|l|}{ Lower jaw } \\
\hline $6-12$ & 17 & 68 & 0 & 0 & 0 & 0.0 & 15 & 60 & 0 & 0 & 0 & 0.0 & 0.0 \\
\hline $13-20$ & 8 & 32 & 0 & 0 & 0 & 0.0 & 20 & 80 & 0 & 2 & 2 & 2.5 & 1.8 \\
\hline $21-25$ & 63 & 252 & 0 & 0 & 0 & 0.0 & 133 & 532 & 2 & 9 & 11 & 2.1 & 1.4 \\
\hline $26-35$ & 12 & 48 & 0 & 0 & 0 & 0.0 & 37 & 148 & 1 & 0 & 1 & 0.7 & 0.5 \\
\hline $36-45$ & 51 & 204 & 0 & 0 & 0 & 0.0 & 101 & 404 & 11 & 7 & 18 & 4.5 & 3.0 \\
\hline $46+$ & 31 & 124 & 0 & 0 & 0 & 0.0 & 48 & 192 & 0 & 1 & 1 & 0.5 & 0.3 \\
\hline Total & 182 & 728 & 0 & 0 & 0 & 0.0 & 354 & 1416 & 14 & 19 & 33 & 2.3 & 1.5 \\
\hline \multicolumn{14}{|c|}{ Upper jaw + lower jaw } \\
\hline $6-12$ & 28 & 112 & 0 & 0 & 0 & 0.0 & 28 & 112 & 0 & 0 & 0 & 0.0 & 0.0 \\
\hline $13-20$ & 21 & 84 & 0 & 0 & 0 & 0.0 & 36 & 144 & 0 & 2 & 2 & 1.4 & 0.9 \\
\hline $21-25$ & 115 & 460 & 0 & 1 & 1 & 0.2 & 246 & 984 & 3 & 9 & 12 & 1.2 & 0.9 \\
\hline $26-35$ & 33 & 132 & 0 & 0 & 0 & 0.0 & 71 & 284 & 1 & 1 & 2 & 0.7 & 0.5 \\
\hline $36-45$ & 81 & 324 & 0 & 0 & 0 & 0.0 & 177 & 708 & 12 & 6 & 18 & 2.5 & 1.7 \\
\hline $46+$ & 56 & 224 & 0 & 0 & 0 & 0.0 & 87 & 348 & 0 & 1 & 1 & 0.3 & 0.2 \\
\hline Total & 334 & 1336 & 0 & 1 & 1 & 0.1 & 645 & 2580 & 16 & 19 & 35 & 1.4 & 0.9 \\
\hline
\end{tabular}


Table 8 Croatian archaeological sites with available paleodental data.

\begin{tabular}{|c|c|c|c|c|c|c|c|c|c|c|c|c|}
\hline $\begin{array}{l}\text { Archaeological } \\
\text { site }\end{array}$ & $\begin{array}{l}\text { Vinkovci - } \\
\text { Gepid }\end{array}$ & Privlaka & $\begin{array}{l}\text { Bijelo } \\
\text { Brdo }\end{array}$ & Suhopolje & Stenjevec & Lobor & Dakovo & Delekovec & Ščitarjevo & Vinkovci & Nova Rača & $\begin{array}{l}\text { Torčec- } \\
\text { Cirkvišče }\end{array}$ \\
\hline Century & $6-7$ & $8-9$ & $10-11$ & 11 & $10-13$ & $11-12$ & $11-13$ & $11-14$ & $11-14$ & $11-14$ & $14-17$ & $14-18$ \\
\hline \multirow{2}{*}{$\begin{array}{l}\text { Number of } \\
\text { examined teeth }\end{array}$} & 248 & 1978 & 979 & 95 & 516 & 135 & 389 & 273 & 45 & 124 & 765 & 73 \\
\hline & $\begin{array}{l}\text { Number of } \\
\text { individuals } \\
\text { (\%) }\end{array}$ & $\begin{array}{l}\text { Number of } \\
\text { individuals } \\
\text { (\%) }\end{array}$ & $\begin{array}{l}\text { Number of } \\
\text { individuals } \\
\text { (\%) }\end{array}$ & $\begin{array}{l}\text { Number of } \\
\text { individuals } \\
\text { (\%) }\end{array}$ & $\begin{array}{l}\text { Number of } \\
\text { individuals } \\
\text { (\%) }\end{array}$ & $\begin{array}{l}\text { Number of } \\
\text { individuals } \\
\text { (\%) }\end{array}$ & $\begin{array}{l}\text { Number of } \\
\text { individuals } \\
\text { (\%) }\end{array}$ & $\begin{array}{l}\text { Number of } \\
\text { individuals } \\
\text { (\%) }\end{array}$ & $\begin{array}{l}\text { Number of } \\
\text { individuals } \\
\text { (\%) }\end{array}$ & $\begin{array}{l}\text { Number of } \\
\text { individuals } \\
\text { (\%) }\end{array}$ & $\begin{array}{l}\text { Number of } \\
\text { individuals } \\
\text { (\%) }\end{array}$ & $\begin{array}{l}\text { Number of } \\
\text { individuals } \\
\text { (\%) }\end{array}$ \\
\hline \multicolumn{13}{|l|}{ Age group } \\
\hline $6-12$ & $5(15.2)$ & $14(8.4)$ & $7(8.6)$ & - & $10(13.5)$ & - & $1(3.3)$ & $1(5.3)$ & $2(22.2)$ & $2(14.3)$ & $\begin{array}{l}36 \text { individuals: } \\
0-15 \text { years } \\
(34.6)\end{array}$ & - \\
\hline $13-20$ & $6(18.2)$ & $17(10.2)$ & $5(6.2)$ & - & 11 (14.9) & $2(22.2)$ & $2(6.7)$ & $1(5.3)$ & $4(44.4)$ & $2(14.3)$ & & $2(15.3)$ \\
\hline $21-25$ & $3(9.0)$ & $23(13.8)$ & $30(37.0)$ & $1(25.0)$ & 9 (12.1) & $2(22.2)$ & $9(30.0)$ & $2(10.5)$ & - & $2(14.3)$ & & 1 (7.7) \\
\hline $26-35$ & $10(30.3)$ & $56(33.5)$ & $8(9.9)$ & $1(25.0)$ & $21(28.4)$ & - & $11(36.7)$ & 6 (31.6) & $1(11.2)$ & $5(35.7)$ & $\begin{array}{l}68 \text { individuals: } \\
15+\text { years } \\
(65.4)\end{array}$ & $3(23.1)$ \\
\hline $36-45$ & $4(12.1)$ & $38(22.8)$ & $23(28.4)$ & $1(25.0)$ & $16(21.6)$ & $2(22.2)$ & $3(10.0)$ & $6(31.6)$ & - & $1(7.1)$ & & $4(30.8)$ \\
\hline $46+$ & $5(15.2)$ & 19 (11.3) & $8(9.9)$ & $1(25.0)$ & 7 (9.5) & $3(33.4)$ & $4(13.3)$ & $3(15.7)$ & $2(22.2)$ & $2(14.3)$ & & $3(23.1)$ \\
\hline Total & $33(100.0)$ & $167(100.0)$ & $81(100.0)$ & $4(100.0)$ & $74(100.0)$ & $9(100.0)$ & $30(100.0)$ & $19(100.0)$ & $9(100.0)$ & $14(100.0)$ & $104(100.0)$ & $13(100.0)$ \\
\hline $\begin{array}{l}\text { Average age at } \\
\text { death (years) }\end{array}$ & 28.6 & 31.2 & 30.1 & 36.5 & 29 & 30.6 & 35 & 34.6 & 24.3 & 28.3 & & \\
\hline $\begin{array}{l}\text { Prevalence of } \\
\text { antemortem } \\
\text { tooth loss (\%) }\end{array}$ & 2.3 & 14.0 & 6.7 & 19.4 & 16.7 & 11.0 & 8.0 & 12.3 & 39.3 & 5.3 & 10.9 & 26.4 \\
\hline $\begin{array}{l}\text { Caries } \\
\quad \text { prevalence (\%) }\end{array}$ & 3.2 & 11.0 & 9.5 & 21.1 & 13.2 & 6.7 & 6.2 & 11.7 & 20.0 & 10.5 & 9.4 & 12.3 \\
\hline \multicolumn{13}{|l|}{$\begin{array}{l}\text { Location of } \\
\text { caries (\%) }\end{array}$} \\
\hline Approximal & 62.5 & 67.1 & 63.4 & 58.8 & 44.4 & 71.4 & 41.7 & 92.9 & 100.0 & 45.5 & 68.0 & - \\
\hline Occlusal & 25.0 & 23.1 & 15.8 & 11.8 & 48.9 & 14.3 & 41.7 & 7.1 & 0.0 & 36.4 & 18.5 & - \\
\hline Buccal & 0.0 & 9.1 & 18.3 & 29.4 & 6.7 & 0.0 & 16.6 & 0.0 & 0.0 & 18.1 & 12.0 & - \\
\hline Lingual & 12.5 & 0.7 & 2.5 & 0.0 & 0.0 & 14.3 & 0.0 & 0.0 & 0.0 & 0.0 & 1.5 & - \\
\hline Total & 100.0 & 100.0 & 100.0 & 100.0 & 100.0 & 100.0 & 100.0 & 100.0 & 0.0 & 100.0 & 100.0 & - \\
\hline $\begin{array}{l}\text { Skeletal root caries } \\
\text { index (SRCI) }\end{array}$ & 0.0 & 0.9 & 0.9 & 0.8 & 1.1 & 0.4 & 0.0 & 0.4 & 0.0 & 0.4 & 0.4 & - \\
\hline
\end{tabular}


Table 9 Comparation of the Bijelo Brdo population with their contemporaries in Italy, Scotland and Turkey.

\begin{tabular}{lcccc}
\hline Archaeological site & Bijelo Brdo - Croatia & La-Selvicciola - Italy & Whithorn - Scotland & Iznik - Turkey \\
\hline Number of individuals & 81 & 48 & 35 & 367 \\
Number of examined teeth & 979 & 912 & 459 & 5709 \\
Prevalence of antemortem & 6.7 & 18.2 & 7.6 & 7.0 \\
tooth loss (\%) & 9.5 & 12.6 & 7.0 & 10.9 \\
\hline
\end{tabular}

noted between the frequencies of carious tooth surfaces and dental roots between anterior $(0.1 \%)$ and posterior teeth $(1.4 \%)$.

Table 8 shows 10 Croatian archaeological sites with available palaeodental data dated in the early and late Middle Ages. All of the sites were located in continental Croatia, and provide palaeodental data for a period of eight centuries. Taking into consideration the size of the samples, the most relevant data were those from the sites of: Privlaka, Nova Rača, Bijelo Brdo, Stenjevac, Vinkovci-Gepid, Dakovo and Delekovec. The average life-span in these sites varied from 28.6 years (Vinkovci-Gepid) to 34.6 years (Delekovec). ${ }^{4}$ The lowest frequency of antemortem tooth loss was recorded in the Vinkovci-Gepid ${ }^{4}$ site $(2.3 \%)$, the highest in Stenjevec ${ }^{4}$ $(16.7 \%)$. The same distribution applies to the frequency of caries. Caries frequencies were lowest in Vinkovci-Gepid (3.2\%), and highest in the sample from Stenjevec ${ }^{4}$ (13.2\%). With the exception of Stenjevec, in all of the analysed samples carious lesions were most frequently recorded interproximally, while frequencies of occlusal caries varied from site to site. SRCI values varied from 0.0 recorded in samples from Vinkovci-Gepid and Dakovo, to 1.1 in the sample from Senjevec. ${ }^{4}$

Table 9 compares data from Bijelo Brdo to data from early mediaeval sites in Italy, Scotland, and Turkey. The Iznik ${ }^{2}$ site in Turkey is by far the largest and best preserved of these sites. Antemortem tooth loss was lowest in the sample from Bijelo Brdo $(6.7 \%)$, and highest in the site of La-Selviccioli ${ }^{9}$ (18.2\%). The population of La-Selviccioli also exhibited the highest frequency of caries (18.2\%), while the lowest frequency was recorded in the population from Whithorn ${ }^{6}$ (7.0\%).

\section{Discussion}

The Bijelo Brdo culture group, named after the eponymous site in Bijelo Brdo near Osijek (eastern Croatia), evolved from the older avaro-slavic culture dated from the 7th-9th century, and existed continuously from the 10th to the 12th century. Archaeological sites belonging to the Bijelo Brdo culture group have been found in eastern and north- ern Croatia, Bosnia and Herzegovina, Austria, Hungary, Slovakia, Serbia, and Romania. ${ }^{12}$

Data obtained by the study of pathological changes in the dental systems of mediaeval populations serve as important resources for evaluating life conditions of our ancestors. ${ }^{6,13-22}$ One of the basic questions that needs to be answered in order to conceive an idea about the quality of life of ancient peoples in a particular historic and geographical frame, is what these people did for a living. The means of earning a living, or in the case of ancient population the means of surviving, determine all other fragments needed to reconstruct their life. The further we go in to the past, the more the dental system offers us in terms of answering a wide spectrum of questions related to the life of a particular community. Skeletal remains of the dental system are a source of information on food, illnesses, tools, social stratification within the community, rituals, as well as age at the time of death.

Systematic bioarchaeological reconstruction of the life and health of ancient populations that inhabited the region of present Croatia had not been conducted until the end of the 20th century. Šlaus analysed bioarchaeological data from 21 sites in continental Croatia, dated from $5000 \mathrm{BC}$ to the 16th century. ${ }^{4}$ Among other things, the frequency of alveolar pathologies (periodontal and periapical abscesses, and antemortem tooth loss), the frequency, distribution, and localisation of caries, as well as tooth enamel hypoplasia, have been noted in this work. Additional data is available from the skeletal remains from the mediaeval cemetery near Suhopolje (Croatia), which belongs to the Bijelo Brdo culture group and is dated to the first third of the 11th century. Boljuncic and Mandic ${ }^{23}$ have analysed the pathological characteristics of the cranium, including the dental system. The data obtained from the mediaeval site of Nova Raca (14th-17th century), analysed by Šlaus and associates $^{24}$ offer additional information on the frequency, distribution, and characteristics of caries, and the pathology of alveolar bone.

The skeletal remains studied in this research were excavated from the mediaeval cemetery at the site of Bijelo Brdo near Osijek during archaeological excavations conducted at the end of the 
19th and the beginning of the 20th century. At first, the remains were stored in the local archaeological museum, and were moved to the Museum of Archaeology in the 1990s. More than half of the total sample was in a state of good or very good preservation (Table 1). The state of preservation was determined on the basis of the condition of each jawbone. For that reason, every kind of estimation is considered to be quite credible. Manzi and associates determined the state of preservation by dividing the total number of teeth present (alveolar cavity) with the total number of teeth, which they got by multiplying the total number of individuals with 32 (number of teeth, i.e. number of alveolar cavities of permanent dentition). ${ }^{9}$ This method is not recommendable because these data do not give a realistic picture about the state of preservation of the sample, because of the possibility that a smaller number of individuals have completely preserved alveolar ridges, while the rest of the sample do not. Such an imbalance can show a non-realistic excellent or poor state of preservation.

The distribution of individuals according to age groups depends on the average life span of the population. The average life span reflects numerous socio-economic factors. ${ }^{9}$ The harder the living conditions are, the shorter the life span. In ancient populations with longer life spans, the frequency and distribution of caries, and antemortem tooth loss increases. In contrast, in contemporary populations, with relatively speaking extremely long life spans when compared to past populations, there is generally a high frequency of antemortem tooth loss, and a low frequency of caries. The population from Bijelo Brdo analysed in this research had an average life span of approximately 30 years and showed an increase of caries and antemortem tooth loss proportional with age. Such a pattern is typical in ancient population as there was no adequate dental care in the past. ${ }^{25}$ The lack of adequate dental care leaves the carious tooth in danger of being lost premortem. ${ }^{6}$

The validity of the direct comparation of the frequency and distribution of caries in medieval populations in Croatia and Europe could be deteriorated because there were several investigators involved. Although the caries diagnostic criteria were well defined (a lesion was considered a caries if there was a clear defect in tooth tissue), there was always a subjective factor which could cause differences between investigators. Therefore such comparisons must be treated with reserve.

The dental sample studied in this research $(979$ teeth of permanent dentition) is one of the largest studied in continental Croatia from the period of the Middle Ages. That, and the high level of preservation contribute to the credibility of the collected data
(Table 8). In cases where the number of recovered dental samples in a particular population are small, false high or low values are possible. In this paper such samples are represented by series from Suhopolje, ${ }^{23}$ Lobor, and Scitarjevo ${ }^{4}$ (the sample consists of four and nine individuals). It is best to exclude such data from the analysis. ${ }^{6}$ Comparing the distribution within particular age groups in samples from Croatian sites, it can be noticed that the number of individuals belonging to the older age groups (older than 25 years) is higher than the number of individuals belonging to the younger (up to 25 years) age groups in nearly all sites.

The data presented in Table 8 covers continental Croatia through a period of eight centuries. The large temporal span, and relatively small area under observation with stabile climatic, hydrological, and geological characteristics enable vertical chronological analysis of the dental illnesses of the people who inhabited this area in different historic periods. Taking into consideration the time period of eight centuries and its logically accompanying improvement in living and work conditions, an increase in the number of carious teeth, and antemortem tooth loss is to be expected because of longer average life-spans. However, while the average life spans of the populations that inhabited Croatia from the 6th to the 14th century, showed a tendency of prolongment, this prolongation was not followed with the expected increase in the number of carious teeth, or antemortem tooth loss. This is not unusual and has been recorded in studies that focused on the dental health of archaeological populations from southwest Scotland, ${ }^{6}$ England, ${ }^{13}$ and Italy, ${ }^{9}$ and can be explained by changes in nutrition. Substances that dominate in a particular nutrition also affect the localisation of caries. In populations whose nutrition is based on solid food, which extensively abrades teeth, higher frequencies of interproximal caries are recorded. Due to abrasion, the occlusal surface is worn out and smoothed and is no longer a predilection spot for caries development. Since the height of the occlusal surface and crown is lowered by abrasion, a compensatory physiological growth of the abraded tooth, up to contact with his antagonist takes place. This leads to greater exposure of interproximal and root surfaces, which become new predilection spots for caries. Therefore, it is considered that there is reduced contribution of interproximal caries in the total frequency of caries in modern populations. Similarly trends could be noticed in Table 8 . if we extract sites with the largest number of studied teeth. Such change of distribution of carious lesions suggests that younger populations consumed softer, less abrasive food, making the occlusal surfaces predilactial spots for caries development. 
Comparing the oral health of the Bijelo Brdo population with that of their contemporaries in Italy, ${ }^{9}$ Scotland, ${ }^{6}$ and Turkey ${ }^{2}$ (Table 9) we notice (with the exception of Italy) relatively uniform frequencies and distribution of caries and antemortem tooth loss. The population of Bijelo Brdo has the lowest frequency of antemortem tooth loss $(6.7 \%)$, and a moderate frequency of caries (9.5\%). Increase in the number of caries is related to an increase in carbohydrate consumption and changes in the cooking and preparation of food. Also, caries frequencies depend on the economy of the society. ${ }^{26}$ Low caries frequencies are found in fishing, hunting and gathering communities $(0.0-5.3 \%)$, while high caries frequencies are recorded in agricultural communities (2.3-26.5\%). Intermediate frequencies $(0.44-10.3 \%)$ are recorded in mixed communities that lived from hunting, as well as agriculture. During the mediaeval period the nutrition of lower socio-economic classes was based on grains and bacon, while consumption of proteins was insignificant. ${ }^{9}$ Taking into consideration the frequency and distribution of caries in Bijelo Brdo it would appear that they pursued both agriculture and hunting, but that their nutrition was primarily grain based, which contributes to the primitive type of caries recorded and the moderate wear out of occlusal surfaces. ${ }^{27}$ The primitive type of caries is characterised by the high frequency of carious cavities on the cemento-enamel junction. It is recorded in populations dated from the Iron Ages $(500 \mathrm{BC})$ to the Middle Ages (1500 AD).

Analysis of the frequency of caries, with respect to type of tooth, enables us to notice a uniform mesiodistal gradient increase in the number of caries from the incisors to the molars. The incisors have the lowest $(1.1 \%)$, while the molars have the highest frequency of caries $(25.3 \%)$. The sample from the site of Whithorn in Scotland, ${ }^{6}$ dated to the same historic period, shows an almost identical gradient with a minor change in the pattern of caries frequencies in the molars - in this series the highest frequency of caries is recorded in the second, followed by the third, and first molar. An identical trend in the frequency of caries with an increase from anterior to posterior teeth is noted in the sample from Aberdeen in Scotland, ${ }^{28}$ dated to the 14 th century. Since the first molar is the first to erupt it is expected to have the highest frequency of caries, but this study shows differently. The first molar would most likely exhibit the highest frequency of caries if we were to know the reason for each case of antemortem tooth loss. However, the analysis of skeletal remains can only determine the fact that a tooth is lost antemortem and not the reason for its loss, i.e. whether the reason for its loss was caries. For that reason, the first molar statis- tically does not have the highest frequency and distribution of caries. Even though this manner of collecting data is the most acceptable one, it can result in contradictions such as this one - that the tooth, which is exposed to the carious effects for the longest period of time and is most probably lost because of caries, does not show the highest frequency of caries.

With respect to the localisation of caries in the sample from Bijelo Brdo, interproximal caries were most frequently recorded. Like the sample of Whithorn, ${ }^{6}$ dated to the same period, occlusal caries is the most frequent in the younger age groups, while in older age groups interproximal caries and caries at the cemento-enamel junction increases significantly. In younger individuals the cemento-enamel junction is covered with the gingiva while in older individuals, due to alveolar absorption and tooth growth because of abrasion the cemento-enamel junction is exposed to caries. ${ }^{28}$

This research showed that the frequency and distribution of caries in a mediaeval population from eastern Croatia were very similar to those of other European populations of the same socio-economic status and nutrition dated to the same historic period. Chronological changes in the localisation of caries are also noted. The proportion of carious lesions, which result from abrasive nutrition, is reduced with time. The collected data also suggest that during the analysed period of eight centuries no significant changes in nutrition occurred. The late medieval populations do not exhibit significantly higher frequencies of caries and higher antemortem tooth loss when compared to populations from the early mediaeval period.

In order to follow the history of dental illnesses of the populations of today's Croatia in terms of different geographical areas, and in chronological terms, it is necessary to study other sites. We recommend that the standards set by Šlaus ${ }^{4}$ additionally modified or expanded in accordance with existing studies conducted in Europe and the rest of the world be adopted for future analyses.

\section{References}

1. Hillson S. Recording dental caries in archaeological human remains. Int J Osteoarchaeol 2001;11:249-89.

2. Duyar I, Erdal YS. A new approach for calibrating dental caries frequency of skeletal remains. J Comparat Hum Biol 2003;54:57-70.

3. Olsson G, Sagne S. Studies of caries prevalence in a medieval population. Dentomaxillofac Radiol 1976;5:12-8.

4. Ślaus M. The bioarcheology of Continental Croatia. BAR 2002.

5. Waldron HA. Are plague pits of particular use to palaeoepidemiologists? Int J Epidemiol 2001;30:104-8. 
6. Watt ME, Lunt DA, Gilmour WH. Caries prevalence in the permanent dentition of a mediaeval population from the south-west of Scotland. Arch Oral Biol 1997;42: 601-20.

7. Lukacs JR. Dental paleopathology: methods for reconstructing dietary patterns. In: Iscan MY, Kennedy KAR, editors. Reconstruction of Life from the Skeleton. New York: Alan R. Liss Inc.; 1989 p. 261-86.

8. Whittaker DK, Molleson T. Caries prevalence in the dentition of a late eighteenth century population. Arch Oral Biol 1996; 41:55-61.

9. Manzi G, Salvadei L, Vienna A, Passarello P. Discontinuity of life conditions at the transition from the Roman imperial age to the early middle ages: example from central Italy evaluated by pathological dento-alveolar lesions. Am J Human Biol 1999; 11:327-41.

10. Beeley JG, Lunt DA. The nature of the biochemical changes in softened dentine from archeological sites. J Archeol Sci 1980;7:371-7.

11. Loveyoj CO. Dental wear in the Libben population: its functional pattern and role on the determination of adult skeletal age at death. Am J Phys Anthropol 1985;68:47-56.

12. Demo Ž. Katalog izložbe "Vukovar - lijeva bara". Zagreb: Arheološki muzej, 1996.

13. O'Sullivan EA, Williams SA, Wakefield RC, Cape JE, Curzon $M E$. Prevalence and site characteristics of dental caries in primary molar teeth from prehistoric times to the 18th century in England. Caries Res 1993;27:147-53.

14. Barthelemy I, Telmont N, Crubezy E, Rouge D. Stomatologic and maxillofacial pathology in a medieval population (10th12th centuries) of southwestern France. Rev Stomatol Chir Maxillofac 1999;100:133-9.

15. Varrela TM. Prevalence and distribution of dental caries in a late medieval population in Finland. Arch Oral Biol 1992; 36:553-9.

16. Pahl KP. Stomatological studies of the slavic cemetery at the Spandau Burgwall, Berlin. Anthropol Anz 1979;37:79-86.
17. Malinowski A. Variability of tooth diseases among the population of Polish territories in the past. Arch Hist Med (Warsz) 1980;42:463-77.

18. Cechova L, Titlbachova S. Tooth status in the population of Prague from the 10th to the 12th century. Cesk Stomatol 1978;78:214-7.

19. Molnar S, Molnar I. Observations of dental diseases among prehistoric populations of Hungary. Am J Phys Anthropol 1985;67:51-63.

20. Djuric Srejic M. Dental paleopathology in a Serbian medieval population. Anthropol Anz 2001;59:113-22.

21. Roulet JF, Ulrich-Bochsler S. Dental examination of skulls of the early Middle Ages in Bial-Mett. SSO Schweiz Monatsschr Zahnheilkd 1979;89:526-40.

22. Sobkowiak EM, Berg P, Held M, Schumacher. Comparative studies of caries epidemiology using skulls from the Iron, Slavic, and Middle Ages as well as modern subjects. Zahn Mund Kieferheilkd Zentralbl 1978;66:359-66.

23. Boljunčić J, Mandić Z. Antropološka analiza kosturnih ostataka iz srednjevjekovnog groblja Zvonimirovo kod Suhopolja (Hrvatska). Prilozi instituta za arheologiju u Zagrebu 1993; 10:131-48.

24. Šlaus M, Pećina-Hrnčević A, Jakovljević G. Dental disease in the late medieval population from Nova Rača, Croatia. Coll Antropol 1997;21:561-72.

25. Marthaler TM. Dentistry between pathology and cosmetics. Community Dent Oral Epidemiol 2002;30:3-15.

26. Hobdell MH, Oliveira ER, Bautista R, Myburgh NG, Lalloo R, Narendran $S$, et al. Oral diseases and socio-economic status (SES). Br Dent J 2003;194:91-6.

27. Vodanović M, Brkić H, Demo Ž., Šlaus M. Dental disease and dietary pattern in the early medieval population from Bijelo Brdo - East Slavonia, Croatia. Acta Stomatol Croat 2003; 37:386-7.

28. Kerr NW, Bruce MF, Cross JF. Caries experience in the permanent dentition of late mediaeval Scots (1300-1600 a.d.). Arch Oral Biol 1988;33:143-8. 